\title{
Survivre à la grippe : exemples concrets des inégalités en santé en temps de pandémie au Canada
}

— Citation : CMAJ 2020 June 22;192:E688-9. doi: 10.1503/cmaj.201074-f; diffusion hâtive le 3 juin 2020

Entrevue avec l'auteur (en anglais) en baladodiffusion : https://soundcloud.com/cmajpodcasts/201074-medsoc

Voir la version anglaise de l'article ici : www.cmaj.ca/lookup/doi/10.1503/cmaj.201074

es éclosions de maladies infectieuses ont toujours mis en évidence les inégalités sociales. Il est clair que la maladie à coronavirus 2019 (COVID-19) ne fait pas exception, et même si les historiens ne se risquent pas à prédire l'avenir, il semble qu'encore une fois, les inégalités influenceront la façon de vivre la pandémie dans le monde. En effet, comme la grippe dite " espagnole » de 1918-1920, la COVID-19 ne frappe pas tout le monde de la même façon.

L'histoire montre que l'expérience vécue durant une épidémie varie selon des facteurs comme la confiance à l'endroit des soins de santé, l'accessibilité de ces derniers, l'existence de filets sociaux et financiers justes et équitables, et la satisfaction des besoins fondamentaux (ex. : accès à l'eau courante potable et sécurité au travail).

Plusieurs historiens ont documenté les disparités sociales durant l'épidémie de grippe de 1918 au Canada. Dans les centres urbains, le taux de mortalité variait considérablement selon le statut socioéconomique, l'ethnicité et la race. Les quartiers défavorisés, y compris ceux où vivaient les nouveaux arrivants, présentaient un taux de mortalité plus élevé dans des villes comme Winnipeg et $H_{a m i l t o n}{ }^{1,2}$. Les décès étaient plus nombreux chez les populations autochtones qu'ailleurs : en effet, la grippe a tué 6,2 Canadiens non autochtones sur 1000, contre 10,3 personnes sur 1000 dans les réserves de l'île-du-Prince-Édouard à 61 sur 1000 dans celles de l'Alberta ${ }^{3}$.

Les chiffres ne révèlent toutefois qu'une partie de l'histoire, car la pandé-

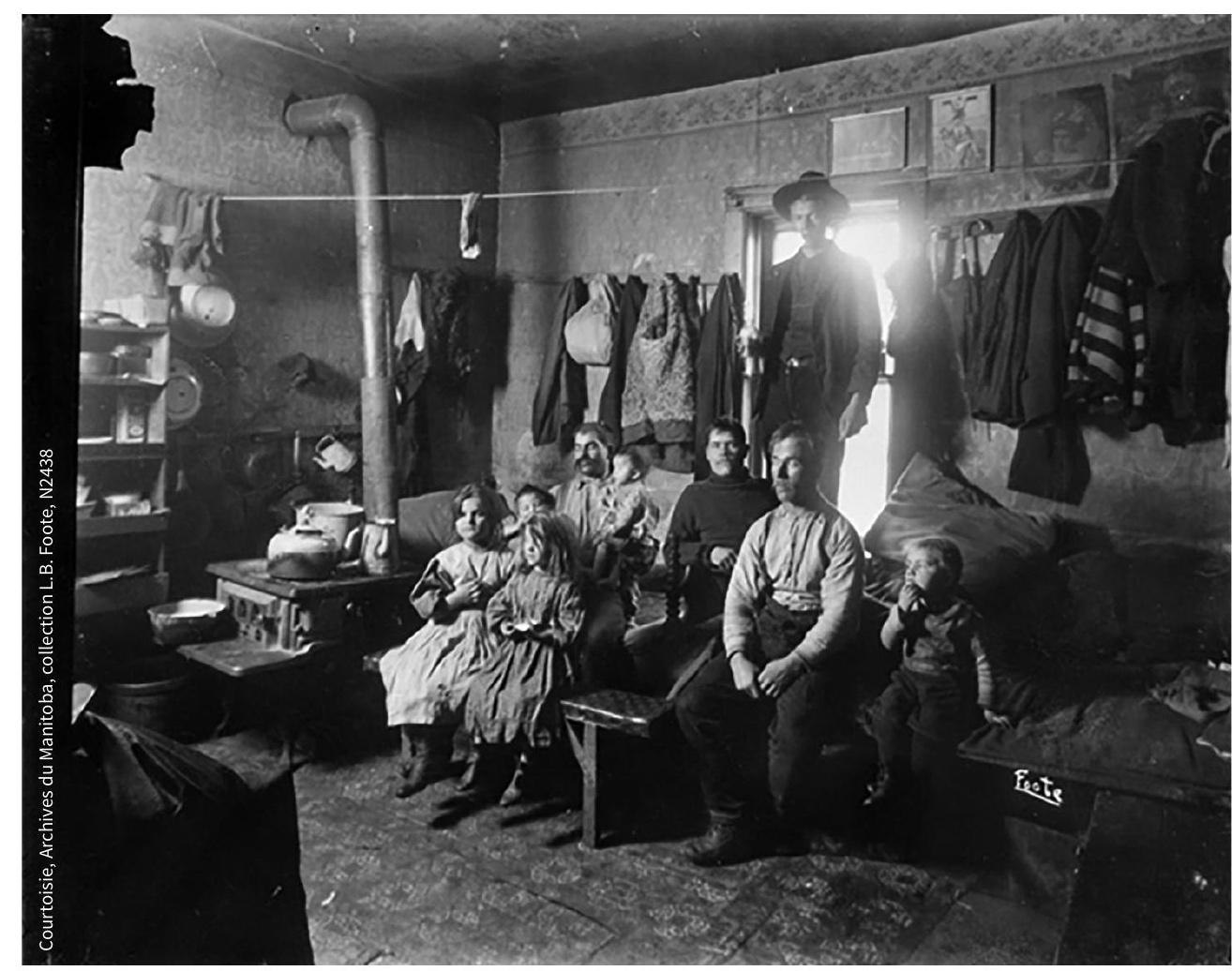

Famille non identifiée (1910).

mie de grippe a aussi eu des effets qualitatifs différents chez les personnes gravement touchées, ce qui a profondément altéré le cours de leur vie. Les personnes mieux nanties n'étaient pas immunisées contre la grippe, mais elles avaient la capacité de lutter contre la maladie, ce qui n'était pas le cas des défavorisées. La grippe s'est rapidement propagée dans les zones urbaines densément peuplées où les familles avaient un accès limité à l'eau chaude courante, où les logements étaient de piètre qualité et mal ventilés et où il était pratiquement impossible d'isoler les cas infectés. Les gouvernements, au courant de la situation et de l'absence d'infrastructures sanitaires dans les quartiers ouvriers, n'avaient pourtant rien fait pour y remédier. S'ils avaient agi, la grippe ne se serait pas propagée aussi rapidement, infectant des familles entières.

À une époque où l'aide sociale n'existait pas encore, les Canadiens faisaient face à un dilemme : continuer à travailler ou ne plus pouvoir payer la nourriture et le loyer. 
Pour ralentir la propagation de la maladie, on a décidé de mettre en quarantaine les malades, sans toutefois leur fournir une aide financière. Les autorités sanitaires craignaient, avec raison, que les gens taisent leurs symptômes afin de pouvoir continuer à travailler. Même aujourd'hui, nous ne connaissons pas toute l'ampleur du problème. Nous savons que les salariés qui s'absentaient pour cause de maladie n'avaient pas droit à des indemnités ce qui est toujours le cas au Canada aujourd'hui - et il n'existait pas d'assurance emploi gouvernementale. Par exemple, les employés des salles de spectacle de Winnipeg, qui avaient perdu plus de 6 semaines de salaire en raison de la fermeture des lieux de loisir, n'ont pas reçu d'aide financière de la part des autorités gouvernementales qui avaient ordonné cette fermeture. Se tournant vers la Ville pour du soutien, leurs épouses et des représentants syndicaux se sont fait répondre que chacun devait faire sa part. C'est ce genre d'attitude qui a attisé les contestations ouvrières et les vagues historiques de grèves du début de 1919.

En 1918-1919, la plupart des Canadiens ont probablement eu du mal à accéder à des soins de santé préventifs ou curatifs durant la pandémie. Le système de santé publique était peu développé, surtout en région rurale. Certaines municipalités rurales (malgré leur rôle important dans l'application des mesures de santé publique à l'époque) ne disposaient pas de responsables de la santé publique ni de régies permanentes en la matière. La plupart des provinces n'avaient pas encore de ministère de la Santé. L'Ontario, par exemple, a créé le sien en 1924 et la ColombieBritannique, en 1946. Le ministère fédéral de la Santé publique n'a vu le jour qu'après la pandémie, en 1919. Pour ce qui est de la prévention, les infrastructures étaient minimales et l'accès aux traitements médicaux, inégalitaire.

Partout au pays, les médecins travaillaient en pratique privée. Bien des gens ne pouvaient pas s'offrir la visite d'un médecin ou d'une infirmière pour recevoir un traitement. Les soins hospitaliers étaient accessibles dans une certaine mesure, mais lacunaires. II n'y avait pas de régime universel d'assurance hospitalisation; la première province à s'en doter a été la Saskatchewan, en 1945. Les hôpitaux religieux et les hôpitaux " publics » en partie subventionnés par les gouvernements sont devenus plus nombreux au début du $20^{\mathrm{e}}$ siècle, mais leurs liens avec la population générale étaient problématiques. La ségrégation à l'égard des minorités raciales et des Premières nations y était fréquente. Par exemple, à l'Hôpital général de Vancouver, les Canadiens d'origine asiatique étaient soignés dans une unité à part, au sous-sol ${ }^{4}$. La même ségrégation raciale existait dans les quelques hôpitaux généraux qui acceptaient les Autochtones : dans l'ensemble, l'accès aux soins de santé leur était limité et inéquitable. Comme l'affirme Maureen Lux dans une récente étude intitulée Separate Beds, "les annexes, ailes et unités au sous-sol destinées aux Autochtones instaurées en réponse aux préjugés de la communauté et inadéquatement financées par l'État, ont activement contribué aux inégalités et au renforcement de l'idée selon laquelle les Autochtones auraient été moins dignes d'être soignés ${ }^{5}$.

Les patients blancs pauvres séjournaient dans des unités beaucoup moins agréables et beaucoup plus achalandées que les patients de la classe moyenne qui avaient les moyens de payer. L'admissibilité au remboursement des frais hospitaliers dans les hôpitaux publics était conditionnelle à un questionnaire indiscret sur les moyens financiers. C'était un processus démoralisant pour les patients, et si les administrateurs hospitaliers jugeaient les familles capables de payer, les soins étaient, paraît-il, souvent refusés.

Tous ces facteurs ont contribué à entretenir une certaine méfiance à l'endroit du système de santé durant la pandémie de grippe de 1918-1920, et pourraient avoir dissuadé les malades de chercher à se faire soigner. Comme lors d'éclosions de maladies infectieuses précédentes, plusieurs victimes de la grippe au Canada ont souffert, ont survécu ou sont décédées à la maison, entourées de leurs proches et de leurs voisins, si elles avaient cette chance. Une certaine solidarité, formelle et informelle, liait les communautés.

La pandémie de grippe de 1918-1920 a été particulièrement éprouvante en raison du profil démographique de ses victimes, qui étaient en majorité de jeunes adultes de 20 à 40 ans. À certains endroits, plus d'hommes que de femmes sont décédés. Les répercussions économiques et émotionnelles pour les enfants et les familles ont été lourdes. Si l'homme pourvoyeur mourait, la famille connaissait une débâcle financière dont elle risquait peu de se remettre. Le problème était exacerbé par les inégalités de genre en matière d'emploi; les femmes avaient moins de choix et recevaient un salaire moindre. Dans certains cas, les mères monoparentales dont les maris étaient décédés de la grippe avaient droit à des prestations, mais seuls le Manitoba et la Saskatchewan offraient de tels programmes en 1918; celui de l'Alberta a été inauguré en $1919^{6}$. Les prestations, maigres, étaient calculées en fonction du revenu des familles et s'accompagnaient de vérifications et de questions incessantes sur la moralité, les aptitudes parentales et les qualités de ménagère de la femme. Au Manitoba, les prestations s'arrêtaient lorsqu'un enfant atteignait l'âge de 14 ans; par conséquent, la plupart ont dû quitter l'école pour travailler. La grippe a donc limité les possibilités d'avenir de toute une génération d'enfants provenant de milieux défavorisés.

Quand les mères mouraient de la grippe, les pères devaient trouver quelqu'un pour s'occuper des jeunes enfants; c'est l'un des facteurs qui a mené à l'augmentation du nombre d'enfants dans les orphelinats juste après la pandémie. Par ailleurs, trop peu d'études analysent la répercussion sociale que constitue l'institutionnalisation des enfants dont la grippe avait emporté un parent ou les deux. À Winnipeg, la Protestant Winnipeg Children's Home a hébergé des enfants des victimes de la grippe, tout comme le Jewish Orphanage, qui a été agrandi pour répondre aux besoins accrus dans l'Ouest du pays ${ }^{7}$. Les orphelinats étaient alors fréquemment utilisés par certains parents pour assurer la sécurité et la survie de leurs enfants, au risque de miner les liens familiaux pendant une intense période de deuil et de pertes.

Après la pandémie, il a fallu du temps au Canada pour se rétablir et retrouver 
des structures sociales normales. En 1919, on a assisté à des manifestations ouvrières partout au pays. Mentionnons plus particulièrement la grève générale de Winnipeg, qui a commencé en mai 1919 et qui a mis la ville sur pause pendant près de 6 semaines. Au cœur de ces luttes : la nécessité d'instaurer un «salaire de subsistance ». L'activisme syndical a cependant été durement réprimé par les gouvernements, qui ont recouru à la force et à l'emprisonnement. Les changements sociaux ne sont survenus que lentement. Lorsqu'ils ont fusionné avec les mouvements ouvriers et agricoles des années 1930, les appels à la réforme des soins de santé se sont inspirés de modèles internationaux de soins de santé socialisés instaurés après la pandémie, y compris de la " médecine communiste » de l'Union soviétique ${ }^{8}$. La naissance de l'assurancemaladie canadienne remonte à ces mouvements en faveur d'une plus grande équité en santé.

\section{Esyllt Jones PhD}

Collège St. John's, Université du Manitoba, Winnipeg, Man.

\section{Références}

1. Jones EW. Influenza 1918: death, disease and struggle in Winnipeg. Toronto: University of Toronto Press; 2007: 59-63.

2. Herring DA, Korol E. The north south divide: social inequality and mortality from the 1918 influenza pandemic in Hamilton, Ontario. In: Fahrni M, Jones EW editors. Epidemic encounters: influenza, society, and culture in Canada, 19181920. Toronto: University of Toronto Press; 2012:97-112.
3. Humphries M. The last plague: Spanish influenza and the politics of public health in Canada. Toronto: University of Toronto Press; 2013:128.

4. Gagan D, Gagan R. "Evil reports" for "ignorant minds"? Patient experience and public confidence in the emerging modern hospital: Vancouver General Hospital, 1912. Can Bull Med Hist 2001;18:349-67.

5. Lux MK. Separate beds: a history of Indian hospitals in Canada, 1920s-1980s. Toronto: University of Toronto Press; 2016: 21

6. Gavigan S, Chunn D. From Mothers' Allowance to mothers need not apply: Canadian welfare law as legal and neo-liberal reforms. Osgoode Hall Law J 2007;45: 741 n 24.

7. Graham S. Building Canadian Jewish citizens out of the abandoned children of Western Canada: the Winnipeg Jewish Orphanage, 1917-1948 [dissertation]. Winnipeg: University of Manitoba; 2020:87.

8. Jones EW. Radical medicine: the international origins of socialized health care in Canada. Winnipeg: ARP Books; 2019.

Cet article a été révisé par des pairs.

Intérêts concurrents : Aucun déclaré. 